\title{
Scaling of Dependence between Foreign Exchange Rates and Stock Markets in Central Europe
}

\author{
L. KRISTOUFEK* \\ Institute of Information Theory and Automation, The Czech Academy of Sciences, \\ Pod Vodarenskou vezi 4, Prague CZ-18208, Czech Republic \\ and Institute of Economic Studies, Faculty of Social Sciences, Charles University in Prague, \\ Opletalova 26, Prague CZ-11000, Czech Republic
}

\begin{abstract}
We propose two novel methodological approaches - the detrending moving average based regression coefficient estimator and the scale-dependent instrumental variable estimator - and show their utility on a specific case of dependence between stock markets and connected foreign exchange rates in the Central European region - the Czech Republic, Hungary, and Poland. The methodology has proven useful as we uncovered several interesting findings such as scale dependence of the shock transmission and differences between the Euro and U.S. dollar currency pairs. The Polish currency is also the most sensitive of the three with respect to the stock market shocks. The proposed methodology can be applied to any system with potential endogeneity issues if one is interested in the scale variability of the effect of interest.
\end{abstract}

DOI: 10.12693/APhysPolA.129.908

PACS/topics: 05.45.Tp, 89.75.Da, 89.65.Gh

\section{Introduction}

Analysis of stock markets and foreign exchange (FX) rates has frequently been a focus of interdisciplinary applications of physics to finance [1-16]. Both stocks and FX rates form a cornerstone of the mainstream financial economics and finance as well due to their tight connection to economic activity of a country (specifically its gross domestic product, GDP) [17-20]. On the one hand, stock markets development has been shown to boost and support economic growth worldwide or, alternatively, the growth of stock markets precedes the economic growth of a referred country [21-23]. On the other hand, economic growth is one of the key macroeconomic variables influencing FX rates of a given country $[18,19]$. Consequently, stock markets and FX rates are intertwined as well. From one side, stock markets growth suggests expanding economy which in turn leads to currency appreciation. From the other side, appreciating currency mitigates profits of stock market foreign investors. The latter relationship is essential for rather small economies whose stock markets are strongly influenced by foreign capital. Here we focus on three such economies - the Czech Republic, Hungary and Poland - which form a majority of the Visegrad Group (less Slovakia which has already adopted the Euro and it is thus not relevant for our analysis), i.e. the group of the Central European post-communist countries. Even though the countries are geographically close, their economic situation and specifically the development of their stock markets varies considerably (ranging from the large Polish market to the quite shallow

\footnotetext{
*e-mail: kristouf@utia.cas.cz
}

Czech market). We analyze the dependence between the local stock markets (specifically the stock indices) and the local currencies. As the relationship between these is complex, the effect may transmit gradually and it may thus be different for various scales (time horizons). To uncover such characteristics, we utilize a regression framework based on the detrended fluctuation analysis and detrending moving average, which is newly introduced here. As an additional methodological novelty, we propose a two-step approach to control for endogeneity between stock indices and FX rates. The expected proportionality between a growing stock market and an appreciating currency is complemented by an uncovered variability across scales for all three analyzed countries.

\section{Methodology \\ 2.1. Model}

We are interested in a basic relationship between a foreign exchange rate (FX) and a stock index (INDEX). Such model can be written as

$$
\mathrm{FX}_{t}=\beta_{0}+\beta_{1} \mathrm{INDEX}_{\mathrm{t}}+\varepsilon_{\mathrm{t}},
$$

where the parameter of interest is $\beta_{1}$, which represents an effect of a change in the stock index on the foreign exchange rate keeping other factors in the error term $\varepsilon$ fixed. However, as argued in Introduction section, a direction of the relationship is not clear and it can, in fact, be bidirectional. This forms a well-known endogeneity problem causing the least squares estimators of $\beta_{1}$ to be biased and inconsistent. As a remedy, the instrumental variable (IV) estimator procedure [24-26] is utilized here. Specifically, we need at least one instrumental variable which is exogenous to FX in Eq. (1) but is correlated with INDEX. Crude oil serves as an ideal candidate as it is tightly connected to stock markets (and even more so in the recent years after the global financial crisis) but 
it does not play a critical role in the FX markets [27]. In turn, we get a complementary regression equation connecting all three variables

$$
\mathrm{INDEX}_{t}=\alpha_{0}+\alpha_{1} \mathrm{FX}_{t}+\gamma_{1} \mathrm{OIL}_{t}+\nu_{t} \text {. }
$$

The IV procedure stems in fitting the right-hand side endogenous variables in Eq. (1), in our case INDEX, with all exogenous variables of our system of equations, in our case OIL. Equation (1) is then rewritten using the fitted values as

$$
\mathrm{FX}_{t}=\beta_{0}+\beta_{1} \widehat{\mathrm{INDEX}}_{t}+\varepsilon_{t} .
$$

The least squares estimator of $\beta_{1}$ is then consistent given the instrumental variable is a good instrument, i.e. it is correlated with $\mathrm{INDEX}_{t}$. In Results section, we show that this is in fact the case. To follow a standard given in the topical literature, we study the logarithmic first differences of the original series to eschew non-stationarity issues and major asymmetries in the analyzed time series. Our procedure then transforms to

$$
\begin{aligned}
& \Delta \log \left(\mathrm{FX}_{t}\right)=\beta_{0}+\beta_{1} \Delta \widehat{\log (\mathrm{INDEX})_{t}+\varepsilon_{t},} \\
& \Delta \log (\mathrm{INDEX})_{t}=\widehat{\alpha}_{0}+\widehat{\alpha}_{1} \Delta \log \left(\mathrm{OIL}_{t}\right) .
\end{aligned}
$$

For the estimation purposes, we utilize the recently proposed detrended fluctuation analysis based regression which allows for disentangling the effect into specific scales. In addition, we introduce a complementary regression estimation based on the detrending moving average procedure.

\subsection{DFA and DMA based regression and estimation specifics}

We shortly recall the necessary steps of the detrended fluctuation analysis (DFA), which is a popular method for describing the fractal nature and long-range dependence properties of time series [28-31]. For time series $\left\{x_{t}\right\}$, a profile is constructed as $X_{t}=\sum_{i=1}^{t}\left(x_{i}-\bar{x}\right)$ which is split into non-overlapping boxes of length $s$ usually referred to as a scale. In a box between $j$ and $j+s-1$, a linear fit of a time trend $\widehat{X_{k, j}}$ is obtained for $j \leq k \leq j+s-1$. Fluctuation function $f_{X}^{2}(s, j)$ is defined for each box of scale $s$ as

$$
f_{X, \mathrm{DFA}}^{2}(s, j)=\frac{\sum_{k=j}^{j+s-1}\left(X_{k}-\widehat{X_{k, j}}\right)^{2}}{s-1} .
$$

The fluctuation $f_{X, \mathrm{DFA}}^{2}(s, j)$ is further averaged over all boxes of length $s$ to get

$$
F_{X, \mathrm{DFA}}^{2}(s)=\frac{\sum_{j=1}^{T-s+1} f_{X}^{2}(s, j)}{T-s} .
$$

For bivariate series $\left\{x_{t}\right\}$ and $\left\{y_{t}\right\}$, the detrended crosscorrelation analysis (DCCA or DXA) $[32,33]$ is based on the generalized fluctuation function

$$
f_{\mathrm{DCCA}}^{2}(s, j)=\frac{\sum_{k=j}^{j+s-1}\left(X_{k}-\widehat{X_{k, j}}\right)\left(Y_{k}-\widehat{Y_{k, j}}\right)}{s-1},
$$

which is again averaged over boxes of length $s$ to find

$$
F_{X Y, \mathrm{DCCA}}^{2}(s)=\frac{\sum_{j=1}^{T-s+1} f_{X Y}^{2}(s, j)}{T-s} .
$$

As the fluctuations $F_{X \text {,DFA }}^{2}(s)$ and $F_{X Y \text {,DCCA }}^{2}(s)$ can be seen as scale-dependent variance and covariance, respectively, these can be further used in correlation and regression analyses [34, 35]. Kristoufek [36] utilizes this correspondence and proposes a new regression framework based on the detrended fluctuation and detrended crosscorrelation analyses that can disentangle the relationship to specific scales. An important advantage over related correlation coefficients $[34,37]$ is the interpretation of the estimated parameter as a direct effect of one variable on the other. Correlation coefficient gives us information only about strength of the relationship but not the actual size of the effect. Specifically, the least squares estimator is rewritten using the scale-dependent variance and covariance so that we have

$$
\widehat{\beta}^{\mathrm{DFA}}(s)=\frac{F_{X Y, \mathrm{DCCA}}^{2}(s)}{F_{X, \mathrm{DFA}}^{2}(s)}
$$

with a use of fluctuations defined in Eqs. (5), (6). More concretely, the DFA-based estimator $\widehat{\beta}^{\mathrm{DFA}}(s)$ is based on a substitution of the scale-dependent variance and covariance into the standard least squares estimator for a simple regression (a more detailed treatment is provided in Ref. [36]). Scale-specific residuals, i.e. deviations of the fitted values $x_{t} \widehat{\beta}^{\mathrm{DFA}}(s)$ from the actual values $y_{t}$, are obtained through

$$
\widehat{u}_{t}(s)=y_{t}-x_{t} \widehat{\beta}^{\mathrm{DFA}}(s)-\overline{y_{t}-x_{t} \widehat{\beta}^{\mathrm{DFA}}(s)} .
$$

The correction $\overline{y_{t}-x_{t} \widehat{\beta}^{\mathrm{DFA}}(s)}$ is necessary here to ensure that residuals have a zero mean and it in fact substitutes the intercept estimation. Using the residuals $\widehat{u}_{t}(s)$, variance of the $\widehat{\beta}^{\mathrm{DFA}}(s)$ estimator is given as

$$
\operatorname{var}\left(\widehat{\beta}^{\mathrm{DFA}}(s)\right)=\frac{1}{T-2} \frac{F_{\widehat{u}, \mathrm{DFA}}^{2}(s)}{F_{X, \mathrm{DFA}}^{2}(s)} .
$$

In the same logic, we can reformulate other methods originally developed for the fractal and long-range dependence analyses to get new estimators for specific scales. As argued by Kristoufek [37, 38], the detrending moving average represents a suitable framework which is comparable to the DFA-DCCA setting on the performance basis but it is also computationally less demanding.

The detrending moving average (DMA) method $[6,39,40]$ is based on the variance scaling with respect to the moving average window length $\lambda$. The method has been further generalized into a bivariate setting [41, 42] and utilized for a construction of new correlation coefficient [37]. Specifically, for time series $\left\{x_{t}\right\}$ and $\left\{y_{t}\right\}$, we construct integrated series $X_{t}=\sum_{i=1}^{t} x_{i}$ and $Y_{t}=\sum_{i=1}^{t} y_{i}$ for $t=1,2, \ldots, T$ where $T$ is the time series length which is common for both series. Fluctuation function $F_{X \text {,DMA }}$ is defined as:

$$
F_{X, \mathrm{DMA}}^{2}(\lambda)=\frac{1}{T-\lambda+1} \sum_{i=\lfloor\lambda-\theta(\lambda-1)\rfloor}^{\lfloor T-\theta(\lambda-1)\rfloor}\left(X_{t}-\widetilde{X_{t, \lambda}}\right)^{2},
$$

where $\lambda$ is the moving average window length and $\theta$ is a factor of the moving average type (forward, centered and backward for $\theta=0, \theta=0.5$ and $\theta=1$, respectively). 
$\widetilde{X_{t, \lambda}}$ represents the specific moving average with the window size $\lambda$ at time $t$. It has been shown [43] that the centered moving average $(\theta=0.5)$ performs the best compared to the other alternatives so that we choose this setting as well.

For the bivariate series, we have the bivariate fluctuation $F_{X Y \text {,DMCA }}^{2}$ (DMCA for the detrending movingaverage cross-correlation analysis) defined as:

$$
\begin{aligned}
& F_{X Y, \mathrm{DMCA}}^{2}(\lambda)=\frac{1}{T-\lambda+1} \sum_{i=\lfloor\lambda-\theta(\lambda-1)\rfloor}^{\lfloor T-\theta(\lambda-1)\rfloor} \\
& \quad \times\left(X_{t}-\widetilde{X_{t, \lambda}}\right)\left(Y_{t}-\widetilde{Y_{t, \lambda}}\right) .
\end{aligned}
$$

Parallel to Eq. (7), we define the DMA-based estimator for a scale $\lambda$ as:

$$
\widehat{\beta}^{\mathrm{DMA}}(\lambda)=\frac{F_{X Y, \mathrm{DMCA}}^{2}(\lambda)}{F_{X, \mathrm{DMA}}^{2}(\lambda)} .
$$

The residuals and variance of the estimator are parallel to the DFA case.

We thus have two estimators of the effect given by the first equation of the system in Eq. (4). This is, however, the second step of the analysis. For the first step, i.e. estimating the second equation of Eq. (4), we use estimators given by averaging either Eq. (7) or Eq. (12) over all analyzed scales. This way, we get a fitted value of the endogenous variable INDEX using the exogenous variable OIL. The procedure is in turn consistent under endogeneity.

\section{Results and discussion}

We analyze the relationship between foreign exchange rates and stock market indices of three Central European countries - the Czech Republic, Hungary and Poland. Specifically, we study the Czech koruna (CZK), Hungarian forint (HUF) and Polish zloty (PLN) as representatives of currencies, and the Prague Stock Exchange PX-GLOB index, Budapest Stock Exchange BUX index and Warsaw Stock Exchange WIG20 index for stock market indices. The analyzed series cover a range between 4.1.2010 and 31.1.2016 (approximately 1500 observations for each country). The crude oil price is represented by the Brent spot prices covering the same period.

The transmission from stock market changes to FX rates is estimated using the two-equation system described in Eq. (4). Specifically, the second equation is fitted using the DFA/DCCA and DMA/DMCA procedures to get fitted values of a relevant stock market index. These are then input into the first equation to avoid endogeneity inconsistency. For the DFA method, we set a minimum scale equal to $10\left(s_{\min }=10\right)$ and a maximum scale equal to $300\left(s_{\max }=300 \approx T / 5\right)$. The system is estimated for each scale between $s_{\min }$ and $s_{\max }$ with a step of 10. Similarly for the DMA method, we set a minimum length of the moving average window to $11\left(\lambda_{\min }=11\right.$, odd numbers are needed due to the central moving averaging), a maximum length is set to $301(\lambda=301 \approx T / 5)$ and a step between scales is set to 10 here as well.
For each pair of the FX rate and stock market, we run a weak instrumental variable test, which stems in testing the instrument significance in the second equation of Eq. (4). For each pair and both the DFA and DMA techniques, the null hypothesis of a weak instrument is rejected at any reasonable significance level, i.e. the $p$-values are well below 0.0001 . Using the crude oil prices thus turns out to be a correct choice.
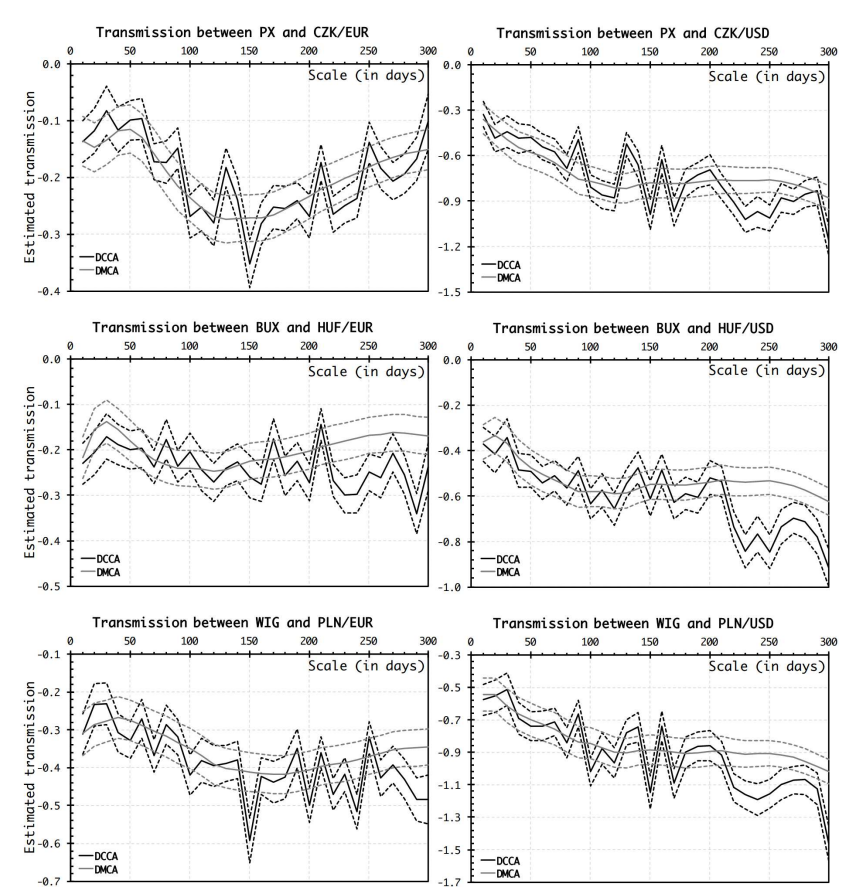

Fig. 1. Relationship between stock market indices and connected FX rates. The rows represent countries - the Czech Republic, Hungary and Poland, respectively - and columns represent the currency pairs the Euro (EUR) and the U.S. dollar (USD), respectively. On the $y$-axes, the estimates (solid lines) and the $95 \%$ confidence intervals (dashed lines) based on DFA (DCCA) are shown in black and the ones based on DMA (DMCA) in gray. The $x$-axes represent scale measured in days.

The resulting effects between stock market indices and FX rates are illustrated in Fig. 1. The rows represent countries - the Czech Republic, Hungary and Poland, respectively - and the columns represent the currency pairs - the Euro (EUR) and the U.S. dollar (USD), respectively. The estimates (solid lines) and the 95\% confidence intervals (dashed lines) based on DFA (DCCA) are shown in black and the ones based on DMA (DMCA) in gray. The estimates based on DFA and DMA (or DCCA and DMCA) are very similar and they overlap in most cases. However, the DMA estimates are much smoother with varying scales. As for the overall findings, there are several common patterns.

Firstly, the estimated effects are negative for all countries, all analyzed scales and both used methods. As the FX rates are defined as the amount of a given local 
currency (CZK, HUF or PLN) for a unit of a global currency (EUR or USD), the negative transmission suggests that an increasing stock market is connected to an appreciating currency. This effect is statistically significant for all analyzed combinations. Secondly, the transmission is below a perfect transmission of -1 for vast majority of cases (we discuss the scale dependence later). Shocks to the stock markets thus do not spill over to the FX markets completely. Thirdly, the effect is higher (in absolute terms) for the USD pairs compared to the EUR pairs. We attribute this to the fact that all three analyzed markets are well integrated into the European Union so that they are mainly affected by common shocks to the whole EU economy rather than local shocks which would transfer more into the EUR exchange rate. Fourthly, the transmission to the EUR pairs is very stable across scales (which is tightly connected to the previous point) with the effect of between -0.2 and -0.4 (the Polish exchange rate reacts to the local stock market shocks more strongly than the Hungarian one). This means that if the stock market increases by a single percentage point, the FX rates appreciate by between 0.2 and 0.4 percentage points. However, this is not true for the CZK/EUR pair for which the transmission is apparently scale dependent. We attribute this to the Czech Nation Bank monetary policy which, starting from November 2013, has kept the CZK/EUR exchange around 27 using monetary interventions. The main motivation behind this move has been to avoid deflation and thus support consumption as well as to boost exports due to a real currency depreciation. Fifthly, the effects for the USD pairs vary strongly with scales. Specifically, the higher the scale (the longer the time horizon) the stronger the effect. For CZK and HUF, the effects vary from approximately -0.3 for the lowest scales up to approximately -1 , i.e. the perfect transmission, for the highest scales (approximately one year). For PLN, the transmission is overall stronger and it varies between approximately -0.6 up to more than -1 . The Polish FX market thus tends to overreact to the stock market shocks in the long term. However, the claim is slightly weakened by the estimates based on DMA which are more conservative. Nevertheless, the Polish FX market is more sensitive to the local stock market shocks than the other two.

\section{Summary}

We have introduced two novel methodological approaches - the DMA-based regression coefficient estimator and the scale-dependent instrumental variable estimator - and have shown their utility on a specific case of dependence between stock markets and connected foreign exchange rates. The methodology has been proven useful as we have uncovered several interesting findings. The proposed methodology can be applied to any system with potential endogeneity issues if one is interested in the scale variability of the effect of interest.

\section{Acknowledgments}

The research leading to these results has received funding from the Czech Science Foundation under project No. 14-11402P.

\section{References}

[1] T. Di Matteo, T. Aste, M. Dacorogna, Physica A 324, 183 (2003).

[2] T. Di Matteo, T. Aste, M. Dacorogna, J. Bank. Fin. 29, 827 (2005).

[3] J. Barunik, L. Kristoufek, Physica A 389, 3844 (2010).

[4] L. Kristoufek, Chaos Solitons Fractals 43, 68 (2010).

[5] L. Kristoufek, Bull. Czech Econom. Soc. 17, 50 (2010).

[6] N. Vandewalle, M. Ausloos, Phys. Rev. E 58, 6832 (1998).

[7] P. Norouzzadeh, B. Rahmanic, Physica A 367, 328 (2006).

[8] T. Mizuno, H. Takayasu, M. Takayasu, Physica A 364, 336 (2006).

[9] M.J. Naylor, L.C. Rose, B.J. Moyle, Physica A 382, 199 (2007).

[10] M. Keskin, B. Deviren, Y. Kocakaplan, Physica A 390, 719 (2011).

[11] J.-P. Onnela, A. Chakraborti, K. Kaski, J. Kertesz, A. Kanto, Phys. Rev. E 68, 056110 (2003).

[12] J.-P. Onnela, K. Kaski, J. Kertesz, Europ. Phys. J. B 38, 353 (2004).

[13] G. Bonanno, G. Caldarelli, F. Lillo, S. Micciche, N. Vandewalle, R. Mantegna, Europ. Phys. J. B 38, 363 (2004).

[14] M. Tumminello, T. Di Matteo, T. Aste, R. Mantegna, Europ. Phys. J. B 55, 209 (2007).

[15] C. Eom, G. Oh, W.-S. Jung, H. Jeong, S. Kim, Physica A 388, 900 (2009).

[16] A. Nobi, S.E. Maeng, G.G. Ha, J.W. Lee, Physica A 407, 135 (2014).

[17] R.E. Bailey, The Economics of Financial Markets, Cambridge University Press, 2005.

[18] P.R. Krugman, M. Obstfeld, M. Melitz, International Economics: Theory and Policy, Prentice Hall, 2014.

[19] P.R. Krugman, M. Obstfeld, M. Melitz, International Trade: Theory and Policy, Prentice Hall, 2014.

[20] F.S. Mishkin, The Economics of Money, Banking and Financial Markets, Prentice Hall, 2015.

[21] R. Levine, Fin. Developm., March 7-10 (1996).

[22] J. Liew, M. Vassalou, J. Fin. Econ. 57, 221 (2000).

[23] S. van Nieuwerburgh, F. Buelens, L. Cuyvers, Explorat. Econ. Hist. 43, 13 (2006).

[24] R.J. Epstein, Oxford Econ. Papers 41, 94 (1989).

[25] J.D. Angrist, G.W. Imbens, D.B. Rubin, J. Am. Statist. Assoc. 91, 444 (1996).

[26] J.H. Stock, F. Trebbi, J. Econ. Perspect. 17, 177 (2003).

[27] C. Baumeister, L. Kilian, CFS Working Paper 501, $1(2015)$ 
[28] C.K. Peng, S.V. Buldyrev, A.L. Goldberger, S. Havlin, M. Simons, H.E. Stanley, Phys. Rev. E 47, 3730 (1993).

[29] C.K. Peng, S.V. Buldyrev, S. Havlin, M. Simons, H.E. Stanley, A.L. Goldberger, Phys. Rev. E 49, 1685 (1994).

[30] C.K. Peng, S.V. Buldyrev, A.L. Goldberger, S. Havlin, R.N. Mantegna, M. Simons, H.E. Stanley, Physica A 221, 180 (1995).

[31] J. Beran, Statistics for Long-Memory Processes, Vol. 61 of Monographs on Statistics and Applied Probability, Chapman and Hall, New York 1994.

[32] B. Podobnik, H.E. Stanley, Phys. Rev. Lett. 100 , 084102 (2008).

[33] W.X. Zhou, Phys. Rev. E 77, 066211 (2008).

[34] G.F. Zebende, Physica A 390, 614 (2011).
[35] B. Podobnik, Z.-Q. Jiang, W.-X. Zhou, H.E. Stanley, Phys. Rev. E 84, 066118 (2011).

[36] L. Kristoufek, Phys. Rev. E 91, 022802 (2015).

[37] L. Kristoufek, Physica A 406, 169 (2014).

[38] L. Kristoufek, Physica A 402, 291 (2014).

[39] E. Alessio, A. Carbone, G. Castelli, V. Frappietro, Europ. Phys. J. B 27, 197 (2002).

[40] A. Carbone, Phys. Rev. E 76, 056703 (2007).

[41] S. Arianos, A. Carbone, J. Statist. Mech. Theory Exp. 3, P03037 (2009).

[42] L.-Y. He, S.-P. Chen, Physica A 390, 3806 (2011).

[43] A. Carbone, G. Castelli, Proc. SPIE 5114, 406 (2003). 